\title{
INFORMATION AND CHOICES IN MATERNITY CARE: A STUDY OF MATERNITY SERVICES IN SOUTH CUMBRIA
}

\author{
Alison Crumbie, Research Officer \\ South Cumbria Community Health Council
}

\section{INTRODUCTION}

The House of Commons Health Committee report (1992)(1) stresses the importance of providing information for women during pregnancy: "unless women are given sufficient, balanced, nonjudgmental and appropriate information at each stage of the maternity process they are unlikely to feel able to make informed choices about their care". Indeed several objectives outlined in the Changing Childbirth report $(1993)^{(2)}$ point to the need for clear unbiased advice to ensure that the woman and her partner feel supported and fully informed throughout pregnancy. Much research has been conducted into information and choices before and after childbirth $^{(3,4,5)}$. Gready, Newburn, Dodds and Gauge ${ }^{(3)}$ found that over half of the women they sampled wished they had been told more during their pregnancy by their doctors or midwives without having to ask, and only $49 \%$ of women who attended hospital antenatal clinics said there was someone there who would listen if they wanted to talk about their pregnancy. Hallgren, Kihlgren, Norberg and Forslin ${ }^{(4)}$ conclude that childbirth education must take perceptions of childbirth into consideration and therefore provision of information cannot be standardised. Fleissig ${ }^{(5)}$ points out that 'feeling in control' and the quality of information received has a direct effect upon the woman's experience of birth and subsequent well-being.

The Community Health Council (CHC) decided to target women and their partners across South Cumbria in the antenatal period. It was accepted that this is a time of change and, therefore, vulnerability and a certain lack of control is to be expected. The focus of the research, however, was on which choices the women had been offered and the information they had been given at each stage. The aim of the research was to determine if women attending antenatal clinics in South Cumbria were fully informed about their choices for antenatal care and delivery.

\section{METHODS}

Discussions were held with midwifery managers at each of the local trusts to determine the most appropriate and effective way to question women in the antenatal period. It was suggested that the consultant's antenatal clinic provided a good opportunity to meet women and their partners. Subsequently, two visits were made to antenatal clinics and the themes of the research were discussed with the women and their partners who were attending on those days. Two questionnaires were developed, piloted and revised. There was one version of the questionnaire for the women and one which was worded slightly differently for their partners. A convenience sample of women and their partners attending the antenatal clinics at Westmorland General Hospital, Furness General Hospital, Dalton Clinic and Ulverston Outpatients Department during a two-week period were handed a questionnaire. This included women who were attending antenatal clinics, midwife booking-in clinics and an antenatal class.

The research officer was in attendance to answer any questions or concerns. Each person who attended was handed a questionnaire. Writing material was provided and a box for questionnaire collection was available at the exit to the waiting room. Each person was given the opportunity to take the questionnaire away for completion at home and a freepost envelope was provided. Over $90 \%$ of the questionnaires were completed whilst the women were waiting for their appointments at the clinic.

\section{RESULTS}

One hundred and thirty-eight questionnaires were handed to women and their partners during the period of the study. The response rate was $95 \%$ and $91 \%$ of the questionnaires were used in the analysis as six of the returned questionnaires were not fully completed.

The sample is a convenience sample and is therefore based on a biased group as it does not include those women who go through their pregnancy without attending the hospital. In discussion with midwifery managers, however, it was estimated at the time of data collection that at least $95 \%$ of pregnant women will at some stage attend the hospital clinic. This sample probably represents quite a large portion of pregnant women in South Cumbria.

The women were asked who first addressed the subject of care during their pregnancy. Fifty-four percent stated that it had been their GP, $23 \%$ had first brought up the subject themselves, and $20 \%$ stated that it was a midwife. Eightynine percent of the women stated that they felt that the timing of the discussion about their care in pregnancy was right.

There is a variety of options for care during pregnancy in South Cumbria. Full care is a term used to describe antenatal care carried out at the hospital by a consultant and the hospital midwives, shared care is care carried out by the GP, midwife and the hospital consultant, GP/midwife care describes care carried out by the GP and midwife at the GP's surgery and midwife only is a relatively new option to women in South Cumbria. 
The women were asked to indicate which choices they had been offered for care during the antenatal period. It was possible to indicate more than one offer. Twenty women had been offered full care, 64 had been offered shared care, nine had been offered $\mathrm{GP} /$ midwife, eight were unsure and seven said they had been offered no choice. Seventy women $(77 \%$ of the sample) had been offered only one form of antenatal care (i.e. no choice). Fifteen had been offered two choices and three had been offered three choices or more.

The women were asked to identify the type of antenatal care that they had chosen. Eleven percent had chosen full care, $65 \%$ shared care, $11 \% \mathrm{GP} /$ midwife care. None had opted for midwife care only and 12 were undecided or had made no decision. Of the 112 respondents who answered this question $95.5 \%$ were satisfied with their decision.

Both women and men were asked what choices they had been offered for delivery. The options in the local area include Furness General Hospital, Westmorland General Hospital, Royal Lancaster Infirmary, home delivery and domino delivery. Figure 1 shows the results. Seventy-four respondents stated that they had been offered only one choice; this represents $63 \%$ of the population. Of the women who answered the question relating to place of delivery $99 \%$ were satisfied with their decision and $92 \%$ of the respondents stated that the timing of the discussion about their plans for delivery was right.

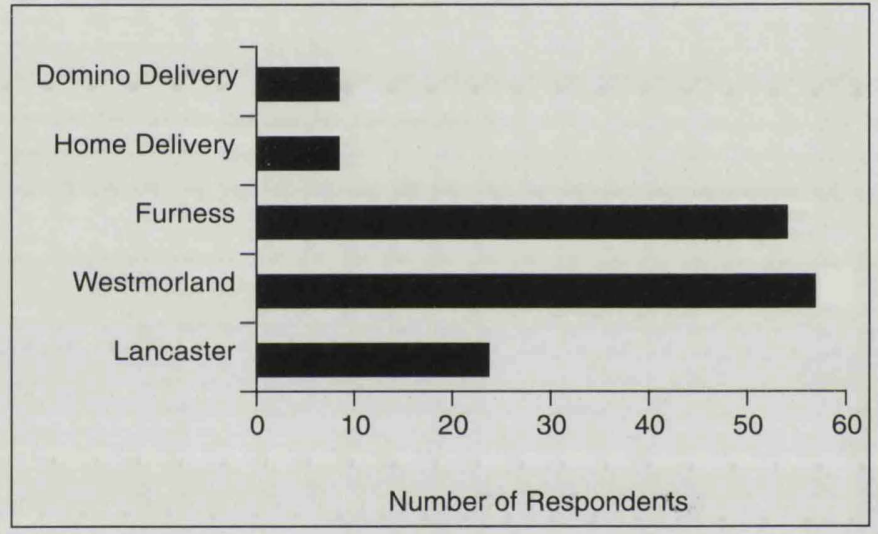

Figure 1 - Choices offered for place of delivery

The women and their partners were asked to identify what had been the most useful method of gaining information about their pregnancy and delivery and Figure 2 summarises the results.

For women, the GP was the most popular source of information, followed by midwives and parenting magazines. Leaflets, friends, the hospital consultant and relatives were all identified as being equally usefuI. Partners rated parenting magazines as more useful than their family doctor or any other source of information.

\section{DISCUSSION AND RECOMMENDATIONS}

It is clear from this study that GPs are a very important contact for women during their pregnancy. The women stated that they found their family doctor most useful in providing information during the antenatal period. A large number of the women sampled, however, were only offered one choice for their care and delivery and were not made aware of the variety of choices available to them. An overwhelming majority of women and their partners were satisfied with the option offered to them and this poses an interesting dilemma for policy makers in South Cumbria.

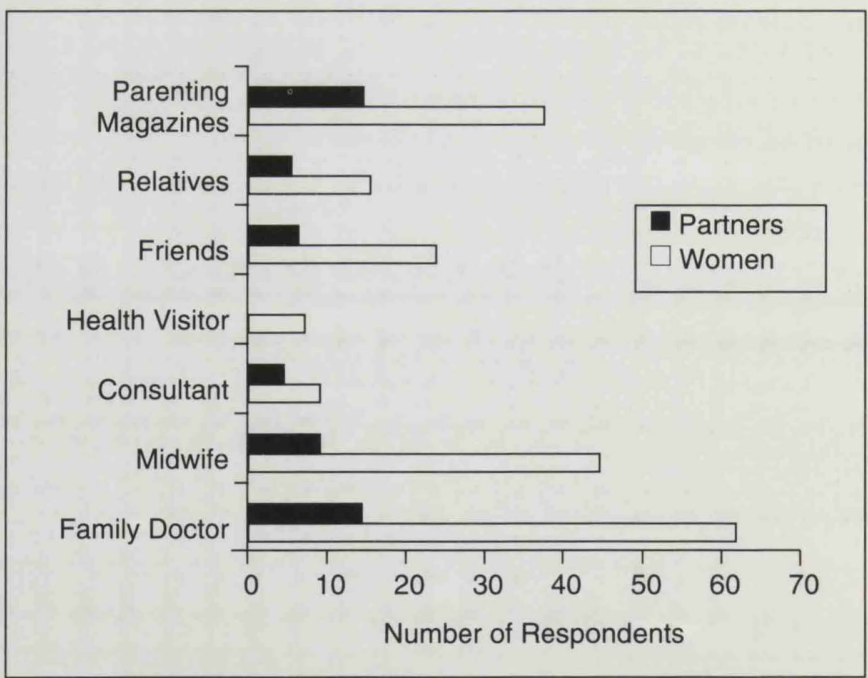

Figure 2 - Most useful method of gaining information

Given that the Changing Childbirth report supports the provision of information and choices for women during pregnancy it would seem to be reasonable that women and their partners should at least know what services are available to them locally. There is a massive amount of information for women and their partners to digest during pregnancy. The quantity and variety of leaflets available and the range of contacts with people who advise on pregnancy and childbirth support this point. It would be unrealistic to expect any one healthcare professional to spend the hours it would take with each woman and/or her partner fully and openly to discuss choices for care and delivery. Yet there is a clear need to be informed and this may explain why so many respondents in this research turned to parenting magazines for their information.

Pregnant women will have different needs and different learning styles, so it would seem appropriate to recommend an eclectic approach to the dissemination of information. Given that resources are inevitably limited, developments for information provision should be planned in an opportunistic and realistic manner. A comprehensive booklet which outlines all the choices and information relating to services offered in the South Cumbria area could be created. GPs and midwives could provide this booklet at the earliest possible opportunity and it could be made available in chemists and other places where a woman might be when she first finds out that she is pregnant.

There also seems to be an ideal opportunity to use an alternative form of information delivery when women are waiting for their appointments in hospital clinics. A variety of educational videos could be created and could be played at all the clinic waiting areas. This may reach some women who learn poorly from written material and may be a reinforcement for those women who have received information from other sources.

The respondents in this survey were very satisfied with the service they were receiving. Providing information about choices for local women during pregnancy could lead to a lower level of satisfaction with the service. When services such as home birth and midwife-only care are not readily available in the local area, is it fair to raise expectations and then be unable to provide those services? The Changing Childbirth report suggests that the local health service should inform women of their choices. It is only when the users of the service are fully informed about what is possible that they can have input to planning developments and improvements for the future of maternity care in South Cumbria. 


\section{REFERENCES}

1 Department of Health, Maternity Services: Government response to the second report from the health committee session 1991-1992. London: HMSO 1992

2 Department of Health Changing Childbirth Part 1: Report of the Expert Maternity Group. London: HMSO 1993

3 Gready M, Newburn M, Dodds R, Gauge S Birth Choices:
Women's Expectations and Experiences. London: National Childbirth Trust 1995

4 Hallgren A, Kihlgren M, Norberg A, Forslin L Women's perceptions of childbirth and childbirth education before and after education and birth Midwifery 1995;11:130-137

5 Fleissig A Are women given enough information by staff during labour and delivery? Midwifery 1993;9:70-75

\section{The Lancaster Medical Book Club Prize}

Please note that it is now time to think about your submissions for the above prize, which is currently worth $£ 100$ to the winner, and is awarded to the author of the adjudged best publication each year.

Candidates must submit a paper in any medical discipline containing original content or application in which he has himself actively participated.

All members of the Lancaster Medical Book Club are eligible individually, or in collaboration, to win the prize, the adjudicators having the discretion to withhold, apportion, or award accumulated income, if necessary.

Submissions should be made to the secretary of the Lancaster Medical Book Club via Lancaster Postgraduate Medical Centre, Ashton Road, Lancaster, LA1 4RR by 1 September annually, to allow judication prior to the Lancaster Medical Book Annual Dinner (usually in late October/early November) where the prize is presented. 